University of Nebraska - Lincoln

DigitalCommons@University of Nebraska - Lincoln

July 1999

\title{
Unoccupied band structure of strained gadolinium
}

\author{
Takashi Komesu \\ University of Nebraska-Lincoln, tkomesu2@unl.edu \\ C. Waldfried \\ University of Nebraska-Lincoln \\ Peter A. Dowben \\ University of Nebraska-Lincoln, pdowben@unl.edu
}

Follow this and additional works at: https://digitalcommons.unl.edu/physicsdowben

Part of the Physics Commons

Komesu, Takashi; Waldfried, C.; and Dowben, Peter A., "Unoccupied band structure of strained gadolinium" (1999). Peter Dowben Publications. 125.

https://digitalcommons.unl.edu/physicsdowben/125

This Article is brought to you for free and open access by the Research Papers in Physics and Astronomy at DigitalCommons@University of Nebraska - Lincoln. It has been accepted for inclusion in Peter Dowben Publications by an authorized administrator of DigitalCommons@University of Nebraska - Lincoln. 


\title{
Unoccupied band structure of strained gadolinium
}

\author{
Takashi Komesu, C. Waldfried, and P. A. Dowben ${ }^{\text {a) }}$ \\ Department of Physics and Astronomy and the Center for Materials Research and Analysis, \\ Behlen Laboratory of Physics, University of Nebraska-Lincoln, Lincoln, Nebraska 68588-0111
}

(Received 2 October 1998; accepted 5 April 1999)

\begin{abstract}
From spin-polarized inverse photoemission, the experimental spin-resolved unoccupied band structure of gadolinium on $\mathrm{Mo}(112)$ has been constructed. The spin-dependent unoccupied electronic structure near the Fermi level is dominated by shallow dispersion of a spin-minority band. A spin-majority and -minority bulk-band pair also exhibits some dispersion across the Brillouin zone. (C) 1999 American Vacuum Society. [S0734-2101(99)17704-2]
\end{abstract}

Gadolinium grown on $\operatorname{Mo}(112)$ exhibits an in-plane expansive strain of about $4 \% .{ }^{1} \mathrm{Gd}(0001)$ thin films grown on $\mathrm{Mo}(112)$ have a band structure quite different from the band structure of gadolinium single crystals and films grown on $\mathrm{W}(110)$ with far less strain. ${ }^{1,2}$ The occupied band structure exhibits a strong wave-vector dependence of the exchangesplitting spin-polarized occupied bands ${ }^{2}$ as well as different band dispersion. With this in mind, we studied the wavevector dependence of the spin-resolved unoccupied band structure of strained thin films of Gd grown on Mo(112) with spin- and angle-resolved inverse photoemission spectroscopy.

Strained thin films of gadolinium of 40-50 ML thickness, with an increased lattice constant of approximately $4 \%$ as compared to $\mathrm{Gd}(0001)$, and a well-ordered hexagonal surface unit cell were obtained by growing $\mathrm{Gd}$ on the corrugated surface of $\mathrm{Mo}(112)$ as described elsewhere. ${ }^{1}$ The wavevector-dependent electronic structure of Gd films of 15-40 ML thickness was studied at $145 \mathrm{~K}$, well below the bulk Curie temperature of $340 \pm 20 \mathrm{~K}$ for these films. The Gd films were magnetized in plane along the substrate corrugation. Spin-polarized inverse photoemission experiments were undertaken with a transversely polarized spin electron gun based upon the Ciccacci design, ${ }^{3}$ as described elsewhere ${ }^{2}$ with a combined resolution of about $390 \mathrm{meV}$ or less. The direction of electron polarization is in the plane of the sample for all incidence angles, as is the applied field. For inverse photoemission, up to six different experiments were summed to improve the signal to noise while insuring that all data are taken immediately following the deposition of the gadolinium. The coercivity of the strained $\operatorname{Gd}(0001)$ films is about 30-50 Oe (depending on film thickness) as determined by magneto-optic Kerr effect (MOKE). ${ }^{2}$ Spin-polarized inverse photoemissions were undertaken in remanance, and since the applied pulsing fields were in excess of $300 \mathrm{Oe}$, the films were saturated after each pulse. Instrumental asymmetry in the electron spectroscopies has been removed by alternating the direction of the field after each sweep of the electron gun energy (spin-polarized inverse photoemission) and summing appropriately to form the spectra.

The spin-polarized inverse photoemission spectra of $\mathrm{Gd}(0001)$ on $\mathrm{Mo}(112)$ are illustrated in Fig. 1, for various

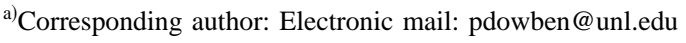

wave vectors along the $\overline{\Gamma \Sigma M}$ symmetry line of the surface Brillouin zone. The Brillouin zone center, at $k_{\|}=0$ or $\bar{\Gamma}$, corresponds to normal incidence in spin-resolved inverse photoemission (unoccupied states). The Brillouin zone edge $\bar{M}\left(k_{\|}=0.96 \AA^{-1}\right)$ requires greater incidence angles according to the relationship between the wave vector parallel to the surface $\left(\underline{k}_{\|}\right)$and the electron incidence angle $(\theta)$ as

$$
\underline{k}_{\|}=\left(2 m / \hbar^{2}\right)^{1 / 2}\left(E_{\text {kin }}\right)^{1 / 2} \sin \theta,
$$

where $E_{\text {kin }}$ is the kinetic energy of the incident electron. As noted before, ${ }^{3}$ both the occupied and unoccupied bands of the strained $\operatorname{Gd}(0001)$ films grown on $\mathrm{Mo}(112)$ are distinct from that of the more "relaxed" films grown on W(110).

Unfortunately, almost all of the bands observed in spinpolarized inverse photoemission exhibit some surface sensitivity. In particular, the spin-majority, spin-minority pair of bands $2-3 \mathrm{eV}$ above the Fermi level (indicated in both Figs. 1 and 2) are extremely sensitive to contamination. In the absence of wave-vector dependence we cannot confirm or reject conservation of two-dimensionality of state for any of the unoccupied bands from our measurements. The exchange-split occupied surface band of $5 d_{z^{2}-r^{2}}, 6 s$ symmetry was observed as two features near the Fermi level: ${ }^{1,2}$ the spin-majority state at $0.7 \mathrm{eV}$ below $E_{F}\left(-0.7 \mathrm{eV} E-E_{F}\right)$ and the spin-minority state at $0.2 \mathrm{eV}$ binding energy $(-0.2$ $\left.\mathrm{eV} E-E_{F}\right)$. The spin minority component of the surface electronic structure may trail across the Fermi level near the zone center $(\bar{\Gamma})$, contributing to the spin minority band above $E_{F}$.

There is a third occupied feature $\Delta_{1}, \Delta_{2}\left(5 d_{z^{2}-r^{2}}, 6 s\right)$ symmetry at $\bar{\Gamma}$ in the valence-band region near $E_{F}$ for strained $\operatorname{Gd}(0001)$ grown on $\operatorname{Mo}(112))^{2,4}$ This is an additional bulk band of majority character, located at approximately $-0.4 \mathrm{eV} E-E_{F}$ at $\bar{\Gamma}$. This bulk band may also actually trail across the Fermi level to the unoccupied side at both $(\bar{\Gamma})$ and $(\bar{M})$ and this is certainly suggested by the spin-polarized inverse photoemission spectra at $\bar{M}$ (Fig. 1), though by no means conclusive. (Such a straddling across the Fermi level of this spin-majority bulk band would act to cancel some of the net polarization of the spin-minority surface state that also may cross the Fermi level, thus leading to little net polarization in our spin-polarized inverse spectra.) The spin-majority bulk band at $0.4 \mathrm{eV}$ binding energy $(-0.4$ $\mathrm{eV} E-E_{F}$ ) of the strained $\mathrm{Gd}$ film on $\mathrm{Mo}(112)$ has a spin 


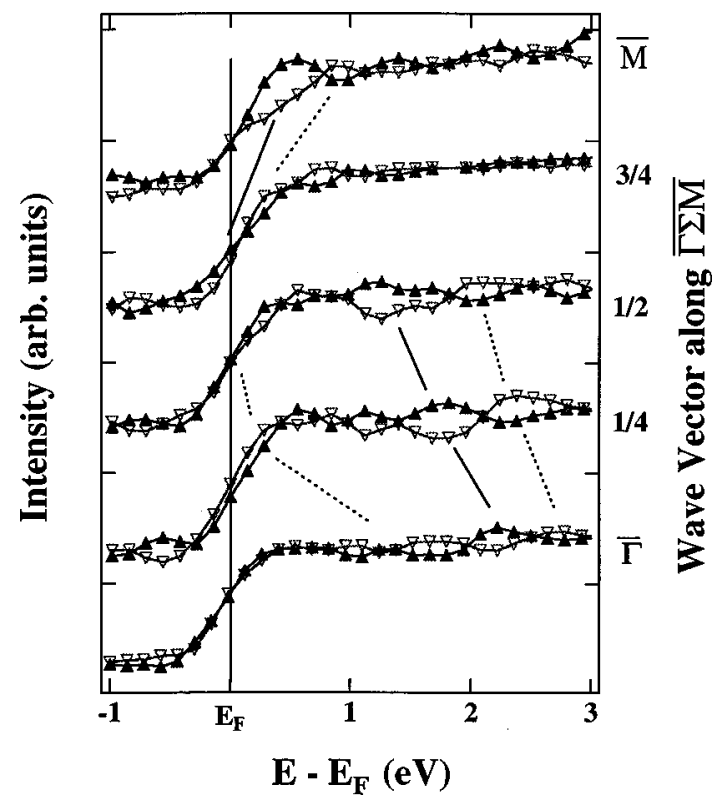

FIG. 1. Spin-polarized inverse photoemission (right) for $\operatorname{Gd}(0001)$ on Mo(112) at approximately $145 \mathrm{~K}$ for various points along the $\overline{\Gamma \Sigma M}$ highsymmetry line from near zone edge $\bar{M}$ to zone center $\bar{\Gamma}$. Spin-majority and spin-minority components are indicated by $(\mathbf{\Lambda})$ and $(\nabla)$, respectively. The spectra were acquired for Gd films of 40 ML thickness.

unoccupied counterpart apparent at about $1 \mathrm{eV}$ to $2 \mathrm{eV}$ above $E_{F}$ at $\bar{\Gamma}$ and $\bar{M}$. The combined spin-polarized photoemission and spin-polarized inverse photoemission indicate an exchange splitting of this bulk band of about $1-1.5 \mathrm{eV}$ at $\bar{\Gamma}^{2,4}$ In the spin-polarized inverse spectra, there is a spin-minority band, with no spin-majority counterpart in the unoccupied band structure at $\bar{\Gamma}$, consistent with this assignment.

The spin-resolved wave-vector-dependent experimental unoccupied band structure from $\bar{\Gamma}$ to $\bar{M}$, along $\overline{\Gamma \Sigma M}$, constructed from angle-dependent spin-resolved inverse photo-

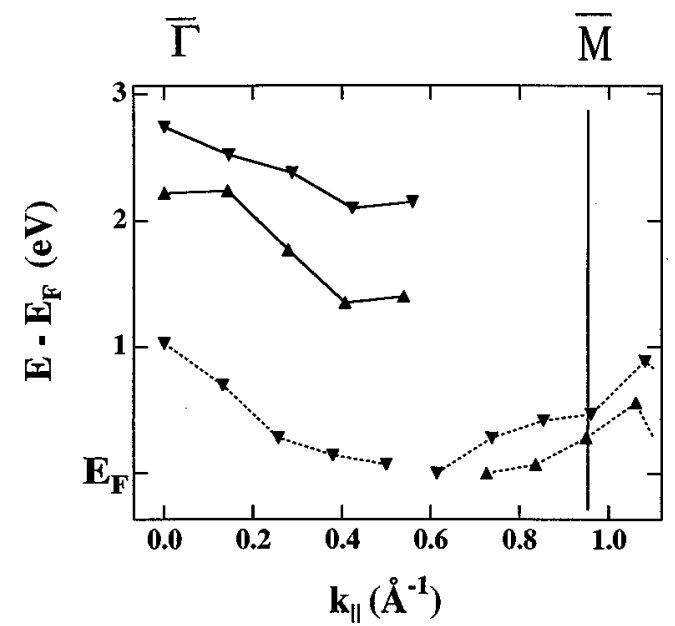

FIG. 2. Spin-resolved unoccupied band structure of $40 \mathrm{ML}$ thick strained Gd films on Mo(112) at approximately $145 \mathrm{~K}$. The filled symbols indicate majority $(\boldsymbol{\Lambda})$ and minority $(\boldsymbol{\nabla})$ bands. The dashed lines indicate that they are postulated to be the components, both majority $(\mathbf{\Lambda})$ and minority $(\boldsymbol{\nabla})$, of bands near the Fermi level (see the text). emission spectra is presented in Fig. 2 for $T / T_{C} \approx 0.5$. The existence of the occupied spin-majority bulk band state of $\Delta_{1}, \Delta_{2}$ symmetry or $6 s, 6 p_{z}$ or $5 d_{3 z^{2}-r^{2}}$ character close to the Fermi level for strained $\operatorname{Gd}(0001)$, across much of the Brillouin zone, will also do much to increase the spinmajority population, at the expense of the spin minority. This majority part of this bulk band straddles the Fermi level with the spin-majority weight observed to be, at least largely, occupied from $\bar{\Gamma}$ to the zone center, ${ }^{2}$ while the spin-minority weight is largely unoccupied at $\bar{\Gamma}$ (Fig. 2).

The effect of the increase in the spin-majority density, particularly so close to the Fermi level both at the surface and in the bulk band structure, may be a significant factor in the increase of the Curie temperature of strained gadolinium grown on $\mathrm{Mo}(112)$ as compared to the unstrained gadolinium. ${ }^{4}$ For the strained $\mathrm{Gd}(0001)$ on $\mathrm{Mo}(112)$, these bands near the Fermi level only weakly disperse and thus are more characteristic of $d$-spectral weight than $s$, suggesting that this could contribute considerable net moment. Strained gadolinium is patently ferromagnetic and this ferromagnetism is reflected in the fact that the unoccupied bulk bands above the Fermi level exhibit a substantial exchange splitting at the zone center (Figs. 1 and 2). The polarization of the bulk bands near and above the Fermi level also demonstrates that there is, indeed, long-range ferromagnetic order. Gadolinium is a local moment system and the origin of the large moment in Gd is the $4 f^{7}$ shallow core level. Coupling occurs through itinerant $5 d, 6 s$ valence electrons, polarized by the $4 f$ moment.

The exchange splittings of bands near and just above the Fermi level are generally observed to be greatest at the $\bar{\Gamma}$ and $\bar{M}$ points within the Brillouin zone reflecting the long- and short-range ferromagnetic order, respectively (Fig. 2). The fully occupied bulk bands well below the Fermi level, by comparison, exhibit no significant exchange splitting at the zone center, indicating the insensitivity of these bands to the long-range ferromagnetic order. ${ }^{2}$ The very large exchange splittings of the bands near the Fermi level compared to other measured exchange splittings for gadolinium thin films on $\mathrm{W}(110)$ are consistent with the enhanced magnetization of the in-plane strained gadolinium on $\operatorname{Mo}(112) .{ }^{5}$ Since these bands are only partly occupied, they contribute to the ferromagnetic order of this system to the greatest extent.

To our knowledge, the spin-polarized inverse photoemission results from strained thin films of Gd is one of the few such studies of the wave-vector-dependent spin-polarized unoccupied band structure in a local moment system like gadolinium. This work provides some indication of the value of spin-polarized inverse photoemission studies in assessing the relationship of the electronic structure on the magnetic properties of an elemental system.

The work was supported by NSF through Grant Nos. DMR-94-07933, DMR-94-96131, and DMR-98-02126. The authors would like to acknowledge conversations with Tim Gay and Markus Donath regarding the spin-polarized inverse photoemission. 
${ }^{1}$ C. Waldfried, D. N. McIlroy, and P. A. Dowben, J. Phys. Condens.: Matter 9, 10615 (1997).

${ }^{2}$ C. Waldfried, T. McAvoy, D. Welipitiya, T. Komesu, P. A. Dowben, and E. Vescovo, Phys. Rev. B 58, 7434 (1998).

${ }^{3}$ F. Ciccacci, H.-J. Drouhin, C. Hermann, R. Houdré, and G. Lampel,
Appl. Phys. Lett. 54, 632 (1989).

${ }^{4}$ T. Komesu, C. Waldfried, and P. A. Dowben, Phys. Lett. A (in press). ${ }^{5}$ C. Waldfried, T. McAvoy, D. Welipitiya, P. A. Dowben, and E. Vescovo, Europhys. Lett. 42, 685 (1998). 